\title{
Orthogonal blocking arrangements for 24-run and 28-run two-level designs
}

\author{
Eric D. Schoen \\ Faculty of Applied Economics, University of Antwerp, Belgium \\ TNO, Zeist, Netherlands \\ Nha Vo-Thanh \\ Faculty of Applied Economics, University of Antwerp, Belgium \\ Institute of Crop Science, University of Hohenheim, Germany
}

\section{Peter Goos}

Faculty of Applied Economics and StatUa Center for Statistics, University of Antwerp, Belgium Faculty of Bioscience Engineering and Leuven Statistics Research Centre (LSTAT), KU Leuven, Belgium

August 28, 2017

\begin{abstract}
Much research has been done concerning 24-run orthogonal two-level designs involving 3-23 factors and 28-run orthogonal two-level designs involving 3-27 factors. The focus of this research was on completely randomized screening designs, and led to lists of recommended designs for each number of factors. When using the recommended designs, there is either no aliasing between the main effects and the two-factor interaction effects or only a limited amount. Among all designs with this attractive property, the recommended designs minimize the aliasing among the two-factor interaction effects. It is, however, unclear which 24- and 28-run designs are best when complete randomization is infeasible and the designs have to be arranged in blocks. In this paper, we address this issue and present the best arrangements of 24-run designs in 3,4 and 6 blocks, and the best arrangements of 28-run designs in 7 blocks.

KEY WORDS: Confounding Frequency Vector; $G$-Aberration; $G_{2}$-Aberration; Generalized Word-Length Pattern; Orthogonal Array; Plackett-Burman Design.
\end{abstract}




\section{Introduction}

Blocking is one of the most important principles of experimental design. To illustrate the relevance of blocking in modern factorial screening experiments, consider the following example. In cheese production, raw milk is processed into curds in large production tanks. Many controllable factors, such as the rotor speed, the amount of rennet and the milk temperature, impact the curds production. Dairy technologists are interested in the effects of nine of these controllable curds production factors on the properties of the cheeses made from the curds. The technologists are primarily interested in the factors' main effects, but they think that a few two-factor interactions might also be active. The traditional type of experimental design to use in that scenario is a two-level orthogonal screening design.

Two-level orthogonal designs are popular experimental plans for screening, because they yield main effect estimates with maximum precision in case a model is estimated with main effects only. The maximum precision is due to the facts that the two-level orthogonal designs are level-balanced (each level of every factor is used equally often) and that the main effect contrast vectors are orthogonal to each other.

A complication when performing the cheese production experiment is that one batch of raw milk allows for at most eight curds to be produced and that substantial batch-to-batch variation may be present. As a result, the required design is a two-level screening design arranged in three blocks of eight runs, where the blocks correspond to the batches of raw milk. Since the budget for the study allows for 24 runs, three blocks of eight experimental runs are required. Note that the original nine factors are generally called the treatment factors, while the batch is generally called the blocking factor of the experiment. Therefore, in the cheese production study, there are nine two-level treatment factors and a three-level blocking factor.

Ideally, the estimates of the treatment factors' main effects are not affected by the batchto-batch or block-to-block variation and the estimation of the two-factor interaction effects is affected as little as possible. We call blocking arrangements for which the main effect 
estimates are not affected by the block-to-block variation orthogonally blocked designs. For such designs, the main effect contrast vectors are orthogonal to the blocks.

Now, it turns out that 71,157,023 different orthogonally blocked 24-run designs exist with nine two-level treatment factors and one three-level blocking factor (Schoen et al., 2010). Based on the research described in this paper, we identified three attractive orthogonally blocked designs, all of which are shown in Table 1 . The orthogonally blocked nature of the designs is due to the fact that the main effect contrast vectors of each of the nine treatment factors sum to zero in each block for each design in Table 1.

For designs 1 and 3 in Table 1, the main effect contrast vectors are not only orthogonal to the blocks, but also to the contrast vectors of the two-factor interactions. Therefore, these designs allow the main effects to be estimated independently from the block effects as well as from the two-factor interactions. Design 2 in Table 1 does not possess this attractive property, but it possesses another interesting characteristic: it allows 14 two-factor interaction effects to be estimated, while designs 1 and 3 only allow 11 two-factor interaction effects to be quantified. Therefore, active interactions might be detected more easily with design 2 than with designs 1 and 3 .

The remainder of the paper is organized as follows. In Section 2, we describe the state of the art when it comes to blocking orthogonal two-level designs and we highlight the contribution of this paper. In Section 3, we review optimality criteria for completely randomized and blocked orthogonal two-level designs. Based on these criteria, we present optimal blocking arrangements of 24- and 28-run designs in Section 4. Next, in Section 5, we describe two real-life experiments in which 24-run designs had to be arranged in blocks. Finally, in Section 6, we discuss our findings and provide a few suggestions for future research.

\section{Literature review and contribution of this paper}

There is a vast amount of literature on blocking two-level designs. Most of it deals with the regular blocking of regular two-level designs. Any $n$-factor regular two-level design consists 


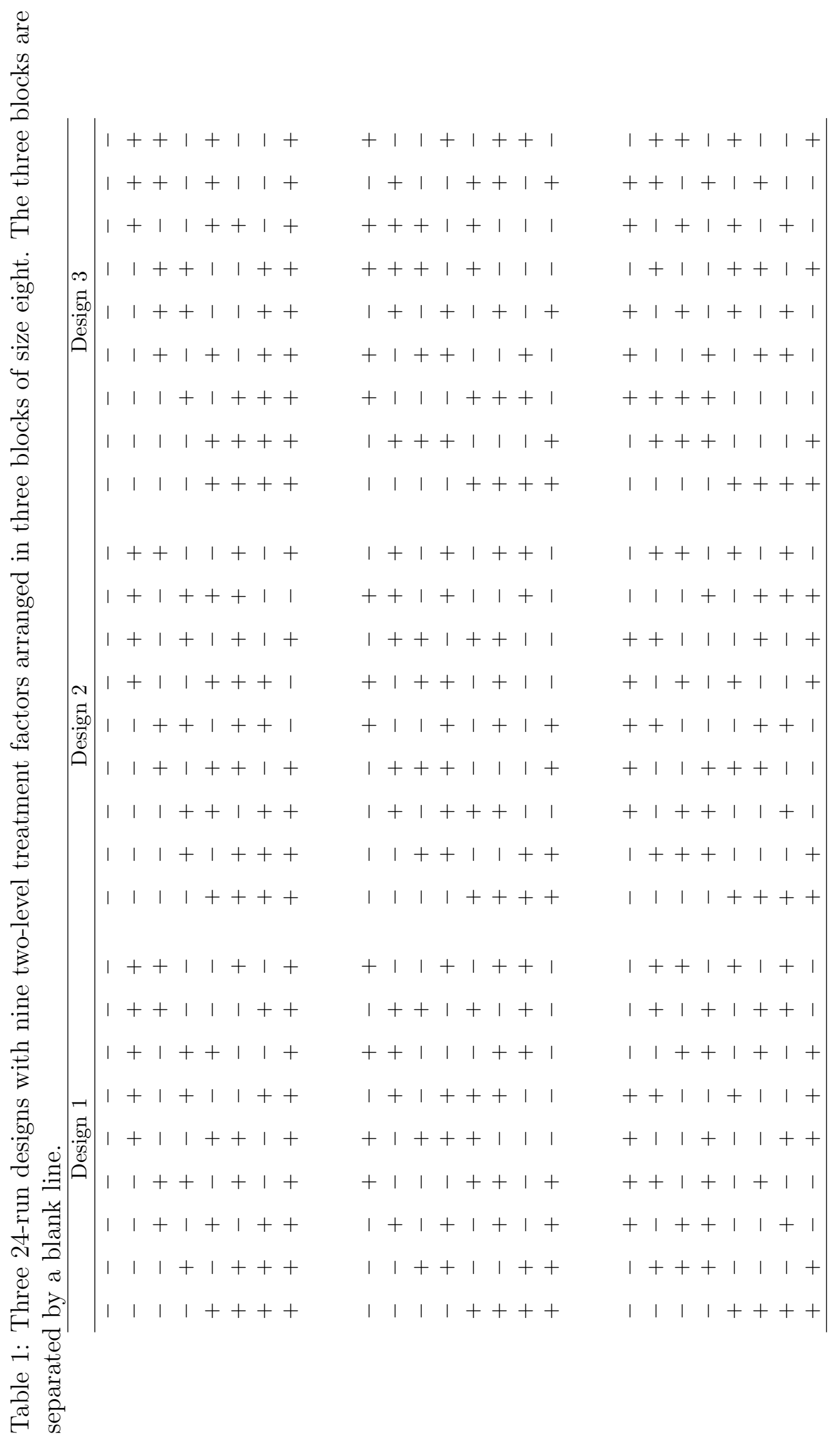


of a $2^{p}$ full factorial design in $p$ basic factors. The settings of the $n-p$ remaining factors are determined by calculating products of the settings of two or more basic factors. Due to this construction, any pair of factorial effects (for instance, main effects and two-factor interactions) is either completely aliased or orthogonal (Montgomery, 2009; Wu and Hamada, 2009), and the run size is a power of two. Regular orthogonal blocking arrangements of regular two-level designs thus have numbers of runs that are powers of two. In addition, the numbers of blocks and the numbers of runs within a block are powers of two as well. Consequently, regular blocking arrangements of regular two-level designs do not offer much flexibility. Another characteristic of the blocking arrangements is that, by construction, the interaction effects are either completely confounded with the blocks or orthogonal to the blocks. So, the estimability of certain interactions is completely sacrificed in these types of blocking arrangements. References discussing these kinds of orthogonal blocking arrangements of regular two-level designs include National Bureau of Standards (1957), Bisgaard (1994), Sun et al. (1997), Sitter et al. (1997), Cheng and Wu (2002) and Xu (2006)

In this paper, the focus is on nonregular orthogonal two-level designs. When using nonregular designs, most pairs of factorial effects are not completely aliased. For this reason, they permit larger numbers of statistical models to be fitted to the data, thereby offering more information on the factorial effects. In addition, when certain interactions are active and a main effects model is estimated, the estimates of the main effect estimates usually have a smaller bias.

Table 2 presents an overview of the literature on nonregular two-level designs, classified according to the run size and the presence or absence of a blocking factor in the designs. The table also positions our work and shows that we fill a gap in the literature by studying the blocking of 24- and 28-run orthogonal two-level designs. The second column of Table 2 indicates the strength of the designs considered in the literature. In strength-2 designs, the main effect contrast vectors are orthogonal. When using strength-3 designs, the main effect 
Table 2: Overview of the literature on nonregular two-level designs and position of the present paper.

\begin{tabular}{cccc}
\hline Run Size & Strength & Without Blocking & With Blocking \\
\hline $12,16,20$ & 2 or 3 & $\begin{array}{r}\text { Deng and Tang (2002) } \\
\text { Li et al. (2004) } \\
\text { Xu and Deng (2005) } \\
\text { Sun et al. (2008) }\end{array}$ & $\begin{array}{c}\text { Cheng et al. (2004) } \\
\text { Schoen et al. (2013) }\end{array}$ \\
\hline 24,28 & 2 or 3 & $\begin{array}{r}\text { Bulutoglu and Ryan (2015) } \\
\text { Schoen et al. (2017) }\end{array}$ & present paper \\
\hline 32,36 & 2 & Schoen et al. (2017) & Schoen (2012) \\
\hline $32,40,48$ & 3 & Schoen and Mee (2012)
\end{tabular}

contrast vectors are not only orthogonal to each other, but also to the contrast vectors of the two-factor interaction effects. In regular two-level orthogonal designs, a strength of 2 corresponds to resolution III, while a strength of 3 corresponds to resolution IV.

All possible nonregular orthogonal two-level designs with up to 24 runs are known and have been studied in detail under the implicit assumption that a completely randomized experiment will be performed and no blocking is required. References for the designs with 12, 16 and 20 runs include Deng and Tang (2002), Li et al. (2004), Xu and Deng (2005) and Sun et al. (2008). Schoen et al. (2017) reviewed the literature on nonregular designs in 24, 28, 32 and 36 runs. Based on the complete enumeration of 24-run designs by Schoen et al. (2010), Schoen et al. (2017) as well as Bulutoglu and Ryan (2015) explored all 24-run orthogonal designs and identified the designs that minimize the amount of aliasing between the main effects and the two-factor interaction effects and, subject to this, the amount of aliasing among the two-factor interaction effects. They also listed the best 28-run nonregular designs involving 3-14 factors and the best 36-run nonregular designs involving 4-18 factors according to these criteria. While Schoen et al. (2017) focused on design exploration, Bulutoglu and Ryan (2015) concentrated on design enumeration algorithms. Finally, Schoen and Mee (2012) explored all strength-3 designs in 32, 40 and 48 runs.

Cheng et al. (2004) were the first authors to propose criteria as well as a search procedure 
to identify good orthogonal blocking arrangements of two-level nonregular designs. Their approach works whenever the number of blocks is a power of two. However, the blocking arrangements shown in Table 1 for the cheese production experiment involve three blocks, and can therefore not be found with the approach of Cheng et al. (2004). Schoen et al. (2013) proposed a more general search procedure for arranging factorial designs of strength 2 in any number of blocks that allows orthogonal blocking. The method, which works for treatment factors with any numbers of levels, is based on complete catalogs of orthogonal designs that include the treatment factors and the blocking factor. Schoen et al. (2013) presented the best blocking arrangements for mixed-level and pure-level designs with 12, 16, 18, 20, and 27 runs. They did not address 24-run and 28-run designs, because the complete catalogs for these designs are very large and a complete exploration of the catalogs was computationally infeasible at the time. Finally, Schoen (2012) presents optimal arrangements of strength-3 designs with 32, 40 and 48 runs in two blocks.

This paper contributes to the literature by presenting optimal arrangements of 24-run designs in three, four, and six blocks and optimal arrangements of 28-run designs in seven blocks. Their run size makes the 24- and 28-run designs a cost-efficient alternative to the strength-3 designs with 32 runs or more. At the same time, they show considerably less aliasing between main effects and two-factor interactions than the strength-2 designs with up to 20 runs. In addition, the two-factor interactions are almost never completely aliased with other two-factor interactions in the 24- and 28-run designs.

\section{Optimality criteria}

In this section, we first review optimality criteria for completely randomized two-level designs. Next, we explain how to quantify the confounding and aliasing in blocked designs. We propose three criteria to deal with blocked orthogonal two-level designs and we illustrate these criteria using the three designs for the cheese making experiment in Table 1. 


\subsection{Criteria for completely randomized designs}

Optimality criteria for two-level orthogonal designs in the absence of blocking include the strength of the design, the rank of the interaction model matrix (which corresponds to a model with an intercept, all main effects and all two-factor interactions; Cheng et al., 2008), $G$-aberration (Deng and Tang, 1999, 2002), and $G_{2}$-aberration (Tang and Deng, 1999).

\subsubsection{Strength}

Orthogonal two-level designs have a strength of $t$ if, for any set of $t$ factors, all $2^{t}$ combinations of factor levels occur equally often. When using strength-2 designs, the main effect contrast vectors are orthogonal. When using strength-3 designs, the main effect contrast vectors are not only orthogonal to each other, but also to the contrast vectors of the two-factor interaction effects. When using strength-4 designs, the contrast vectors of the two-factor interactions are orthogonal to each other too. A consequence of the definition of strength is that any strength- $t$ design has a run size proportional to $2^{t}$. The 24-run designs studied in this paper can therefore either have strength 2 or strength 3. The 28-run designs all have a strength of 2 , because 28 is not a multiple of $2^{3}=8$.

\subsubsection{Rank}

The rank of the interaction model matrix is of interest to researchers who want to identify active two-factor interactions in addition to main effects. The larger the rank, the larger the number of estimable two-factor interactions. For example, the rank of the interaction model matrix of the first and the third design option in Table 1 equals 21. The second option has a rank of 24. The larger rank might be a reason to prefer the second option over the first and the third. 


\subsubsection{G-aberration}

The $G$-aberration criterion is based on counting correlations between contrast vectors. Of particular interest in orthogonal two-level designs are correlations between main effect contrast vectors and two-factor interaction contrast vectors (type-3 correlations) and correlations among the two-factor interaction contrast vectors (type-4 correlations). Deng and Tang (1999) showed that the only possible absolute type-3 and type-4 correlations for orthogonal $N$-run two-level designs equal $(N-8 j) / N$, where $j$ is a non-negative integer smaller than or equal to $N / 8$. So, the only possible absolute type-3 and type- 4 correlations are $1,2 / 3$, $1 / 3$ and 0 for orthogonal 24-run designs, and $1,5 / 7,3 / 7$ and $1 / 7$ for orthogonal 28-run designs. For any given design, the $F_{3}$ vector quantifies the frequencies of the absolute type-3 correlations for pairs of one main effect and one two-factor interaction effect. The $F_{4}$ vector quantifies the frequencies of the absolute type- 4 correlations for pairs of two two-factor interaction effects. More specifically, the $F_{3}$ and $F_{4}$ vectors contain the frequencies of the different type- 3 and type- 4 correlations divided by 3 . The division by 3 for the type- 3 correlations is due to the fact that, for any set of three factors, there are three pairs of one main effect contrast vector and one two-factor interaction contrast vector, all of which have the same correlation. The frequencies for the type- 4 correlations are divided by 3 for a similar reason. Generally, the first entries for the $F_{3}$ and $F_{4}$ vectors correspond to the most severe correlations, while the last entries correspond to the least severe correlations. The frequencies of the zero type- 3 and type- 4 correlations are usually omitted from the $F_{3}$ and $F_{4}$ vectors.

In the notation of the $F_{3}$ and $F_{4}$ vectors, it is customary to explicitly mention the values $N-8 j$ rather than the absolute correlations $(N-8 j) / N$. So, an $F_{3}$ vector for a 24 -run design is denoted by $F_{3}(24,16,8)$ instead of $F_{3}(1,2 / 3,1 / 3)$, while an $F_{3}$ vector for a 28 -run design is denoted by $F_{3}(28,20,12,4)$ instead of $F_{3}(1,5 / 7,3 / 7,1 / 7)$. A similar notation is used for the $F_{4}$ vectors.

For an $n$-factor design, in addition to the $F_{3}$ and $F_{4}$ vectors, we can also define the $F_{5}, \ldots, F_{n}$ vectors involving the frequencies of the different absolute type- $5, \ldots$, type- $n$ cor- 
relations. Together, all these vectors form the confounding frequency vector $\left(F_{3}, F_{4}, \ldots, F_{n}\right)$. A minimum $G$-aberration design minimizes the confounding frequency vector's entries from left to right. The $G$-aberration of a design is its ranking in terms of the confounding frequency vector.

Note that the $F_{5}$ and $F_{6}$ vectors quantify the extent to which three-factor interaction contrast vectors are correlated with two-factor interaction contrast vectors and other three-factor interaction contrast vectors, respectively. In most applications, three-factor interactions are assumed to be negligible. Therefore, it is not particularly interesting to minimize the $F_{5}$ and $F_{6}$ vectors. The same is true for the $F_{7}, \ldots, F_{n}$ vectors, which deal with interactions of an even higher order. Therefore, in this paper, we restrict our attention to the $F_{3}$ and $F_{4}$ vectors. Note that strength-3 designs do not involve nonzero type-3 correlations. Therefore, the elements of their $F_{3}$ vectors all equal 0 .

\subsection{4 $G_{2}$-aberration}

Tang and Deng (1999) proposed a simpler version of the confounding frequency vector called the generalized word length pattern (GWLP). To calculate the GWLP's first element, all type-3 correlations are squared and summed, and the result is divided by 3 . The resulting figure is called the generalized word count of length 3 and denoted by $A_{3}$. Generalized word counts of length $4,5, \ldots, n$ are defined in a similar fashion, and denoted by $A_{4}, A_{5}, \ldots, A_{n}$.

The GWLP is the vector $\left(A_{3}, A_{4}, \ldots, A_{n}\right)$. A minimum $G_{2}$-aberration design minimizes the GWLP's entries from left to right. The $G_{2}$-aberration of a design is its ranking in terms of the GWLP. We restrict our attention to the $A_{3}$ and $A_{4}$ counts for reasons similar to those given in Section 3.1.3. Finally, note that, for strength-3 designs, $A_{3}=0$.

\subsubsection{Illustration}

For designs 1 and 3 in Table 1 , the $F_{3}$ and $F_{4}$ vectors equal $F_{3}(24,16,8)=(0,0,0)$ and $F_{4}(24,16,8)=(0,0,126)$, respectively. Design 2 has $F_{3}(24,16,8)=(0,0,10)$ and $F_{4}(24,16,8)=$ 
$(0,0,98)$. So, when using design 1 or 3 , there are no occurrences of the absolute type- 3 correlations of $1,2 / 3$ and $1 / 3$ between a main effect contrast vector and a two-factor interaction contrast vector. In other words, the designs have strength 3 and all main effect contrast vectors are orthogonal to the two-factor interaction contrast vectors. Moreover, the only occurring absolute type- 4 correlation is $1 / 3$, meaning that every pair of two-factor interaction contrast vectors has a correlation of $+1 / 3$ or $-1 / 3$.

When design 2 would be used, $10 \times 3=30$ pairs of a main effect contrast vector and a two-factor interaction contrast vector would have a correlation of $\pm 1 / 3$. This can be seen from the value of 10 in the $F_{3}$ vector for that design. The remaining pairs of a main effect contrast vector and a two-factor interaction contrast vector would be uncorrelated. The fact that its $F_{3}$ vector is not a zero vector is due to the fact that design 2 only has a strength of 2 .

Starting from the $F_{3}$ and $F_{4}$ vectors, the $A_{3}$ and $A_{4}$ values of the design options in Table 1 can be computed. For designs 1 and $3, A_{3}=0 \times 1^{2}+0 \times(2 / 3)^{2}+0 \times(1 / 3)^{2}=0$, and $A_{4}=0 \times 1^{2}+0 \times(2 / 3)^{2}+126 \times(1 / 3)^{2}=14$. For design $2, A_{3}=1.11$ and $A_{4}=10.89$.

\subsection{Confounding and aliasing in blocked designs}

When screening designs have to be blocked, a primary concern is to ensure that the main effects can be estimated independently from the block effects. For this reason, we restrict our attention to orthogonally blocked designs. The next most important concern involves the confounding of the two-factor interactions with the blocks. By far the most common approach is to minimize the confounding of the two-factor interactions with the blocks. In doing so, it might be easier to detect active interaction effects. Schoen et al. (2013), however, explain that maximizing the confounding between the two-factor interactions and the blocks may be sensible as well. As a matter of fact, this alternative approach would ensure that the mean squared error of the main effects model has the smallest possible upward bias due to active interactions, and would result in a maximum power for detecting active main effects 
when using a model involving main effects only.

Each of the two approaches to deal with two-factor interactions requires measuring the extent to which they are confounded with the block effects. Schoen et al. (2013) quantified the confounding of the two-factor interactions with the blocks by means of the $F A_{3}$ vector, a frequency vector of generalized counts of length-3 words that involve the blocking factor.

To explain the construction of the $F A_{3}$ vector, consider the problem of arranging a twofactor two-level treatment design with 24 runs into three blocks. The first step is to define an orthogonal coding for the three-level blocking factor. One possible choice of coding involves dummy variables $c_{1}$ and $c_{2}$ which take the values -1 and 1 , respectively, for the first block, 0 and -2 for the second block, and 1 and 1 for the third block. The next step is to normalize the contrast vector containing all $24 c_{1}$ and $c_{2}$ values to length $\sqrt{24}$ and to calculate the inner products between the treatment factors' two-factor interaction contrast vector, on the one hand, and the normalized contrast vectors with $c_{1}$ and $c_{2}$ values, on the other hand. Next, the two resulting inner products have to be divided by 24 . This results in two correlations between the interaction contrast vector and the contrast vectors of the three-level blocking factor. Finally, summing the squared correlations produces the generalized word count of length 3 for the $3 \times 2 \times 2$ design involving the three-level blocking factor and the two two-level treatment factors. We denote that generalized word count of length 3 by $A_{3}^{(b 22)}$. Xu and $\mathrm{Wu}(2001)$ show that the $A_{3}^{(b 22)}$ value does not depend on the exact choice of the orthogonal coding scheme for the blocking factor.

The smaller the value of $A_{3}^{(b 22)}$, the less severe the aliasing between the main effect of the three-level blocking factor and the interaction involving the two-level treatment factors. There are four different orthogonal 24-run designs with one three-level factor and two twolevel factors (Schoen et al., 2010). Studying these four designs shows that the possible generalized word counts of length 3 for one three-level factor and two two-level factors are $2 / 3,1 / 2,1 / 6$ and 0 . In other words, the only possible values of $A_{3}^{(b 22)}$ are $2 / 3,1 / 2,1 / 6$ and 0 . Smaller values indicate a smaller degree of confounding between the two-factor interactions 
and the blocks. A zero value for $A_{3}^{(b 22)}$ indicates that the contrast vector of the two-factor interaction is orthogonal to the blocks.

For any orthogonal 24-run design with one three-level (blocking) factor and $n$ two-level (treatment) factors, there exist $n(n-1) / 2$ projections of the design into one three-level factor and two two-level factors. The $F A_{3}$ vector lists the frequencies with which the nonzero $A_{3}^{(b 22)}$ values of $2 / 3,1 / 2$ and $1 / 6$ occur in these projections. For example, the first two (9-factor) designs in Table 1 have an $F A_{3}$ vector of $(0,0,18)$, where the vector's first, second and third entries correspond to the $A_{3}^{(b 22)}$ values $2 / 3,1 / 2$ and $1 / 6$, respectively. This means that 18 of the 36 two-factor interactions have an $A_{3}^{(b 22)}$ value of $1 / 6$ (indicating some confounding of the two-factor interactions with the blocks), while the remaining 18 have an $A_{3}^{(b 22)}$ value of zero (indicating no confounding with the blocks). For the third design option in Table 1 , the $F A_{3}$ vector is $(0,9,18)$. As a result, when that design would be used, nine additional interactions would be confounded with the blocks. The extent of that additional confounding would also be more severe, because of the $A_{3}^{(b 22)}$ value of $1 / 2$ for these nine interactions.

A more concise measure of the confounding between the two-factor interactions and the blocks is the total generalized count of length-3 words involving the blocking factor and the two-factor interaction contrast vectors, which we call the total block count and denote by $A_{3}^{(b t o t)}$. We obtain this count by multiplying the frequencies in the $F A_{3}$ vector with the corresponding generalized word counts and summing the resulting products. For example,

the total block count $A_{3}^{(b t o t)}$ of the first two designs in Table 1 equals $0 \times 2 / 3+0 \times 1 / 2+$ $18 \times 1 / 6=3$, whereas it equals 7.5 for the third design. Obviously, the smaller the block count, the smaller the overall extent to which two-factor interactions are confounded with the blocks.

\subsection{Criteria for optimal blocking}

To identify optimal blocking arrangements, we adapt the criteria proposed by Cheng and $\mathrm{Wu}$ (2002) for regular two-level designs, Cheng et al. (2004) for nonregular orthogonal two-level 
designs and Schoen et al. (2013) for general orthogonal nonregular designs to the specific cases of blocked nonregular two-level designs considered here. We name the resulting criteria the $W_{2}, W_{2}^{-}$and $W_{3}$ criteria and we collectively call them 'the three criteria'. Like the $G$ aberration and $G_{2}$-aberration criteria, these criteria involve a vector that has to be minimized from left to right. The difference between the aberration criteria and our criteria is that our minimization vectors involve mixes of the generalized word counts $A_{3}, A_{4}$ and $A_{3}^{(b t o t)}$, the $F_{3}, F_{4}$ and $F A_{3}$ vectors, and, in one criterion, the rank $R$ of the interaction model matrix. The exact minimization vectors for our design selection criteria are shown in Table 3.

Table 3: Three criteria for selecting optimally blocked two-level designs

\begin{tabular}{ccccccc}
\hline Criterion & \multicolumn{6}{c}{ Minimization vector } \\
\hline$W_{2}$ & $-R$ & $A_{3}$ & $F_{3}$ & $A_{3}^{(b t o t)}$ & $A_{4}$ & $F_{4}$ \\
$W_{2}^{-}$ & $A_{3}$ & $F_{3}$ & $-A_{3}^{(b t o t)}$ & $A_{4}$ & $F_{4}$ & \\
$W_{3}$ & $F_{3}$ & $F A_{3}$ & & & & \\
\hline
\end{tabular}

\subsubsection{The $W_{2}$ criterion}

As can be seen from the first element of its minimization vector in Table $3,-R$, our $W_{2}$ criterion seeks designs with a large rank $R$ for the interaction model matrix, i.e., designs that allow many two-factor interaction effects to be estimated. The minus sign in $-R$ indicates that minus the rank should be minimized, which is the same as maximizing the rank itself. The inclusion of $-R$ as the first element in the $W_{2}$ minimization vector distinguishes our $W_{2}$ criterion from that in Schoen et al. (2013). The modification allows us to detect interesting strength-2 designs (for which $A_{3}>0$ ) with many estimable two-factor interaction effects, while the original $W_{2}$ criterion leads to the selection of strength-3 designs (for which $A_{3}=0$ ) with fewer estimable two-factor interaction effects. However, strength-3 designs will still be optimal in terms of the $W_{3}$ criterion, since any design for which $A_{3}=0$ also has a zero $F_{3}$ vector. In conclusion, by inserting $-R$ in the $W_{2}$ minimization vector, we increase the difference between the $W_{2}$ and $W_{3}$ criteria. This allows us to identify a larger set of useful blocking arrangements. 
Besides $-R$, the elements of the $W_{2}$ criterion's minimization vector include the generalized length-3 word count $A_{3}$ of the two-level treatment factors, the treatment factors' $F_{3}$ vector, the block count $A_{3}^{(b t o t)}$, the generalized length- 4 word count $A_{4}$ of the treatment design, and its $F_{4}$ vector. Positioning the $F_{3}$ vector immediately behind the $A_{3}$ value emphasizes our desire to avoid strong correlations between main effect contrast vectors and two-factor interaction contrast vectors among all design options with the same generalized length-3 word count. For a similar reason, we position the $F_{4}$ vector behind the $A_{4}$ value. Cheng et al. (2004) considered separate criteria for the generalized word counts $A_{i}$ and for the confounding frequency vector (which involves the $F_{3}$ and $F_{4}$ vector). We combine the generalized word counts and the confounding frequency vector to reduce the number of different criteria to consider.

In the $W_{2}$ criterion's minimization vector, the block count $A_{3}^{(b t o t)}$ is preceded by the generalized length-3 word count $A_{3}$ and itself precedes the length- 4 count $A_{4}$. So, minimizing the confounding of the two-factor interactions with the blocks is prioritized over minimizing the aliasing among the two-factor interactions. This makes sense especially when large block effects are anticipated or when the number of blocks is small, in which cases the available inter-block information about confounded interaction effects is very limited and confounding of two-factor interactions with the blocks should be avoided to the largest possible extent.

Several authors also consider a $W_{1}$ criterion, which switches the positions of the block count $A_{3}^{(b t o t)}$ and the generalized length-4 word count $A_{4}$ (Cheng and Wu, 2002; Cheng et al., 2004; Schoen et al., 2013). We believe that this criterion is not useful for the cases with three of four blocks studied here, for the reasons that led us to recommend the $W_{2}$ criterion. Nevertheless, we did search for blocking arrangements that are optimal with respect to the $W_{1}$ criterion. It turned out that these blocking arrangements only differ from the $W_{2}$ optimal arrangements for 7 - and 8-factor 28-run designs in 7 blocks. We therefore only pay attention to the $W_{1}$ criterion for these two cases. 


\subsubsection{The $W_{2}^{-}$criterion}

Schoen et al. (2013) introduced the $W_{2}^{-}$criterion to facilitate the detection of active main effects, by maximizing the confounding between the two-factor interaction effects and the blocks. In this way, the mean squared error of the main effects model would be inflated as little as possible due to any active two-factor interactions, resulting in the largest possible test statistic values for the treatment factors' main effects' significance tests.

Schoen et al. (2013) also discuss a $W_{1}^{-}$criterion, which reverses the prioritization of the maximization of the confounding between two-factor interactions and blocks and the minimization of the length-4 word count $A_{4}$. However, as the aim is to estimate a simple main-effects-only model, prioritizing the maximization of the confounding between twofactor interactions and blocks is more useful than minimizing the aliasing among two-factor interactions. For this reason, we do not use the $W_{1}^{-}$criterion in the present paper.

\subsubsection{The $W_{3}$ criterion}

The $W_{3}$ criterion is based on the $F_{3}$ and $F A_{3}$ vectors. Loosely speaking, it first minimizes the number of two-factor interactions that are severely confounded with the main effects and, subject to this, it minimizes the number of two-factor interactions that are substantially confounded with the blocks. It is, of course, possible to extend the minimization vector with the $F_{4}$ vector. However, for the designs considered in this paper, adding the $F_{4}$ vector to the minimization vector does not lead to different optimal designs.

\subsection{Illustration of the three criteria}

For each of the three blocking arrangements in Table 1 , Table 4 shows the $W_{2}, W_{2}^{-}$and $W_{3}$ criteria's minimization vectors. The second design option in Table 1 outperforms the other two designs in terms of the $W_{2}$ criterion, because it has the interaction model matrix with the largest rank, namely 24. The third design in Table 1 is best according to the $W_{2}^{-}$criterion, because it has the largest block count $A_{3}^{(b t o t)}$ of the three designs (7.5), in addition to a zero 
Table 4: The $W_{2}, W_{2}^{-}$and $W_{3}$ criteria's minimization vectors for the three designs in Table 1

\begin{tabular}{cllllll}
\hline$W_{2}$ criterion: & $-R$ & $A_{3}$ & $F_{3}$ & $A_{3}^{(\text {btot })}$ & $A_{4}$ & $F_{4}$ \\
\hline Design 1 & -21 & 0 & $(0,0,0)$ & 3 & 14 & $(0,0,126)$ \\
Design 2 & -24 & 1.11 & $(0,0,10)$ & 3 & 10.89 & $(0,0,98)$ \\
Design 3 & -21 & 0 & $(0,0,0)$ & 7.5 & 14 & $(0,0,126)$ \\
\hline$W_{2}^{-}$criterion: & & $A_{3}$ & $F_{3}$ & $-A_{3}^{(b t o t)}$ & $A_{4}$ & $F_{4}$ \\
\hline Design 1 & & 0 & $(0,0,0)$ & -3 & 14 & $(0,0,126)$ \\
Design 2 & & 1.11 & $(0,0,10)$ & -3 & 10.89 & $(0,0,98)$ \\
Design 3 & 0 & $(0,0,0)$ & -7.5 & 14 & $(0,0,126)$ \\
\hline$W_{3}$ criterion: & & $F_{3}$ & & & $F A_{3}$ \\
\hline Design 1 & & $(0,0,0)$ & & & $(0,0,18)$ \\
Design 2 & & $(0,0,10)$ & & & $(0,0,18)$ \\
Design 3 & & $(0,0,0)$ & & & $(0,9,18)$ \\
\hline
\end{tabular}

$A_{3}$ value and a zero $F_{3}$ vector. Finally, the first design option in Table 1 outperforms the second design option in terms of the $W_{3}$ criterion, because it has a better $F_{3}$ vector. It outperforms the third design option in terms of the $W_{3}$ criterion, because it has the same ideal $F_{3}$ vector and a better $F A_{3}$-vector.

From an exhaustive search over all nonisomorphic designs involving nine two-level (treatment) factors and one three-level (blocking) factor produced by the enumeration algorithm of Schoen et al. (2010), we were able to find out that the designs 1, 2 and 3 in Table 1 are optimal in terms of the $W_{3}$ criterion, the $W_{2}$ criterion and the $W_{2}^{-}$criterion, respectively. The $W_{2}^{-}$optimal design (design 3$)$ involves the same treatments as the $W_{3}$ optimal design (design 1), but the treatments are assigned to the three blocks in a different fashion. This can be seen from the fact that the designs have the same $A_{3}$ and $A_{4}$ values and the same $F_{3}$ and $F_{4}$ vectors, but a different $A_{3}^{(b t o t)}$ value and a different $F A_{3}$ vector.

Which of the three designs is preferred depends on the exact goal of the experimenters. If interactions are considered as nuisance effects, designs 1 and 3 both stand out. Design 3 is preferred if one wants to start with a main effects model and estimate the standard errors based on the mean squared error, while there might be a number of small two-factor interactions. The mean squared error is less biased when using this design than when using the other two designs. Design 1 is preferred if one wishes to remove one or two more 
Table 5: Numbers of nonisomorphic orthogonally blocked two-level designs with 24 and 28 runs

\begin{tabular}{|c|c|c|c|c|}
\hline \multirow{2}{*}{$\begin{array}{l}\text { Number of } \\
\text { factors }\end{array}$} & \multicolumn{3}{|c|}{24 runs } & 28 runs \\
\hline & 3 blocks & 4 blocks & 6 blocks & 7 blocks \\
\hline 2 & 4 & 4 & 4 & 4 \\
\hline 3 & 29 & 25 & 25 & 25 \\
\hline 4 & 573 & 552 & 226 & 371 \\
\hline 5 & 28,745 & 21,757 & 2,663 & 21,502 \\
\hline 6 & $1,089,168$ & 457,768 & 19,323 & 395,598 \\
\hline 7 & $14,576,216$ & $3,113,669$ & 58,112 & $1,422,094$ \\
\hline 8 & $57,436,095$ & $8,168,256$ & 65,679 & $1,005,490$ \\
\hline 9 & $71,157,023$ & $12,605,571$ & 26,454 & 135,569 \\
\hline 10 & $33,893,515$ & $15,119,461$ & 12,243 & 4,296 \\
\hline 11 & $10,266,252$ & $14,961,206$ & 4,882 & 104 \\
\hline 12 & $3,305,030$ & $12,096,092$ & 1,543 & 21 \\
\hline 13 & 981,180 & $7,855,020$ & 277 & \\
\hline 14 & 220,993 & $4,066,838$ & 45 & \\
\hline 15 & 32,567 & $1,665,918$ & & \\
\hline 16 & 2,282 & 532,484 & & \\
\hline 17 & & 129,122 & & \\
\hline 18 & & 22,880 & & \\
\hline 19 & & 2,758 & & \\
\hline 20 & & 238 & & \\
\hline
\end{tabular}

substantial interactions from the mean squared error by including interaction terms in the model. Finally, design 2 is recommended if finding interaction effects is an important goal of the experiment.

\section{Optimal blocking arrangements}

Table 5 shows the numbers of nonisomorphic orthogonal 24-run two-level treatment designs involving a three-level, a four-level and a six-level blocking factor as well as the numbers of nonisomorphic orthogonal 28-run two-level treatment designs involving a seven-level blocking factor (Schoen et al., 2010). By an exhaustive evaluation of all these designs in terms of the three criteria, we were able to identify all optimal orthogonally blocked two-level designs involving 24 or 28 runs. Our exploration of the complete catalog was performed in the same way as the search done by Schoen et al. (2013) for smaller designs. As the numbers 
of nonisomorphic designs are very large, this exploration was computationally demanding. Supplementary materials to this paper include all optimal orthogonally blocked designs with four or more treatment factors, as well as tables showing the $F_{3}, F_{4}$ and $F A_{3}$ vectors along with the ranks of the interaction model matrices for the optimal designs.

We visualize the generalized length-3 word counts $A_{3}$ and the block counts $A_{3}^{(b t o t)}$ of the optimal designs involving four or more two-level treatment factors in Figure 1. Panels (a) and (b) of the figure show results for 24-run designs involving three blocks. Panels (c) and (d) show results for 24-run designs involving four blocks. Panel (e) visualizes the results for 24-run designs involving six blocks, and, finally, panel (f) contains results for 28-run designs and seven blocks.

Every vertical line in the panels corresponds to one optimal blocking pattern we identified. The lower end of each line indicates the generalized length-3 word count $A_{3}$ of the two-level treatment design, while the length of the line indicates the block count $A_{3}^{(b t o t)}$. As a result, a low lower end of a line means a small degree of aliasing between the treatment factors' main effects and their two-factor interactions. A short vertical line means a small degree of confounding between the treatment factors' two-factor interaction effects and the blocks. A high higher end of a vertical line means a severe aliasing of the two-factor interactions with the main effects and/or a severe confounding of the two-factor interactions with the block effects. So, the higher end of each line provides a measure of the total confounding and aliasing of the two-factor interactions, $A_{3}+A_{3}^{(b t o t)}$.

Generally, the vertical lines appear in clusters of three and correspond, from left to right, to the $W_{2}$ optimal, $W_{2}^{-}$optimal, and $W_{3}$ optimal designs for the number of treatment factors displayed on the horizontal axis. Whenever a cluster contains only one or two lines, this means that we identified designs that are optimal with respect to more than one criterion. 


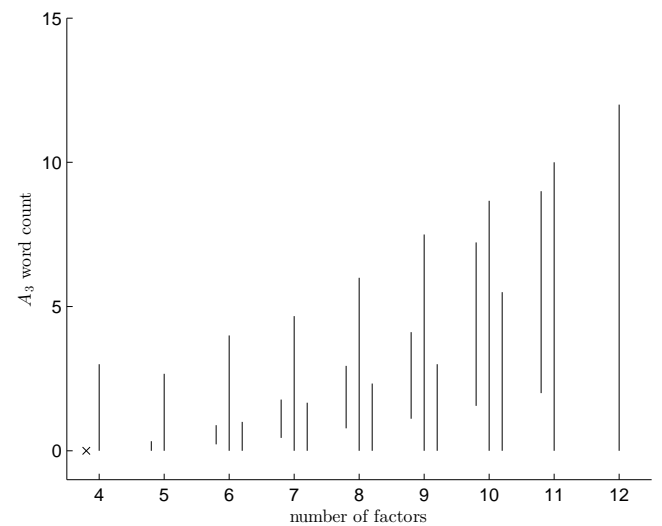

(a) 24 runs, 3 blocks, 4-12 factors

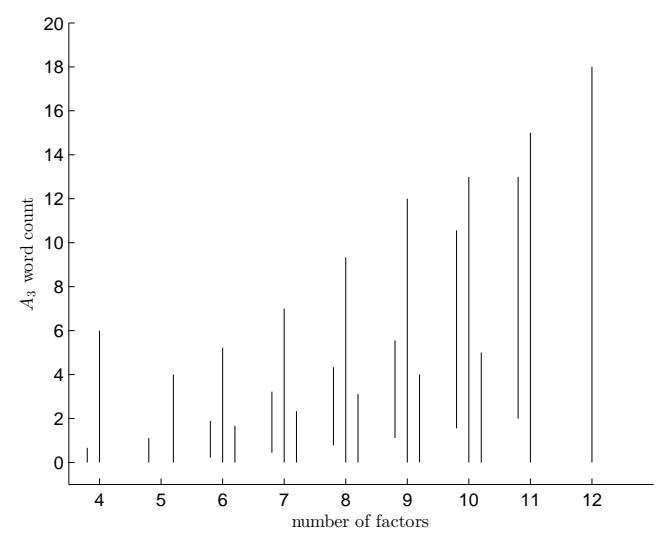

(c) 24 runs, 4 blocks, 4-12 factors

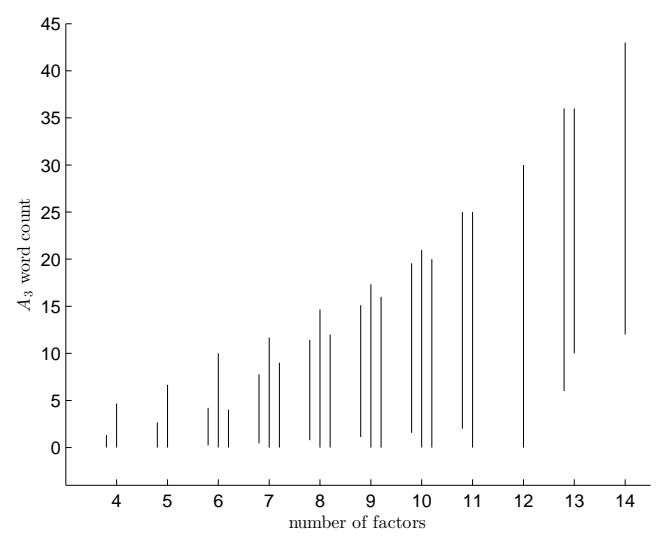

(e) 24 runs, 6 blocks, 4-14 factors

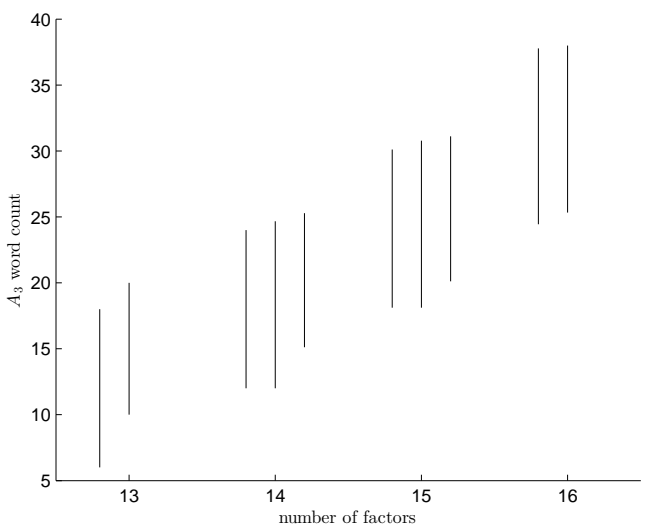

(b) 24 runs, 3 blocks, 13-16 factors

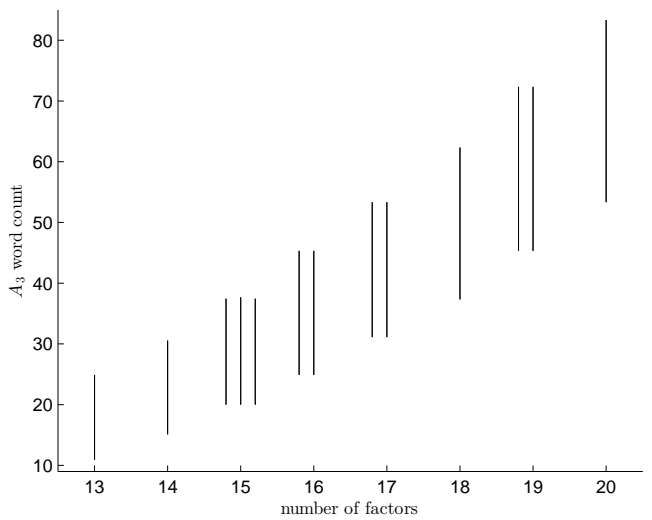

(d) 24 runs, 4 blocks, 13-20 factors

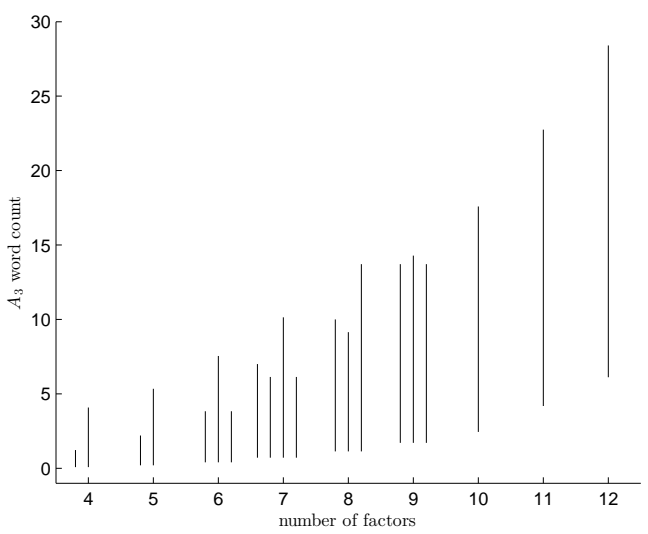

(f) 28 runs, 7 blocks, 4-12 factors

Figure 1: Properties of the optimal blocking arrangements of 24-run and 28-run orthogonal designs. The lower ends of the vertical lines indicate the $A_{3}$ values for the treatment designs, while the lines' lengths represent the block counts $A_{3}^{(b t o t)}$. 


\subsection{4-run designs in three blocks}

Panel (a) of Figure 1 shows the results for 24-run designs involving 4-12 treatment factors and three blocks. For each number of treatment factors, the plot shows one or two designs of strength 3 , which have a generalized length-3 word count, $A_{3}$, of zero. These designs are optimal in terms of the $W_{2}^{-}$and $W_{3}$ criteria, because these criteria prioritize the minimization of the $A_{3}$ value. In all of these cases, the same two-level treatment design is used in the $W_{2}^{-}$ and $W_{3}$ optimal blocked designs.

For the case of nine treatment factors, the strength-3 treatment design, along with its $W_{2}^{-}$ and $W_{3}$ optimal blocking arrangements, is shown in Table 1. Design 3 in Table 1 corresponds to the $W_{2}^{-}$optimal design, while design 1 is the $W_{3}$ optimal design.

All designs involving four or five treatment factors have a strength-3 treatment design. The small cross corresponds to a 4-factor design that does not involve any confounding of the two-factor interactions with the blocks. It consists of two replicates of the regular $2_{I V}^{4-1}$ design and one replicate of that design's foldover. Each of the three replicates is assigned to a different block. The design is $W_{2}$ optimal and $W_{3}$ optimal. The second design with four factors involves three replicates of the regular $2_{I V}^{4-1}$ design. Its runs are assigned to the blocks in such a way that the runs in the first block all have $a+1$ in the contrast vectors for the interaction between treatment factors 1 and 2 and the interaction between treatment factors 3 and 4 , the runs in the second block all have a -1 in the contrast vectors for the interaction between treatment factors 1 and 4 and the interaction between treatment factors 2 and 3 , and the runs in the third block all have a -1 in the contrast vectors for the interaction between treatment factors 1 and 3 and the interaction between treatment factors 2 and 4 . This design is $W_{2}^{-}$optimal. In the optimal blocking arrangements for five treatment factors, two different treatment designs are used. The first one is used in the $W_{2}$ optimal and $W_{3}$ optimal blocking arrangement, while the second is used in the $W_{2}^{-}$optimal blocking arrangement. Unlike the designs with four treatment factors, the five-factor treatment designs cannot be constructed using regular fractions. 
The $W_{2}$ optimal designs involving 6-11 treatment factors have a nonzero generalized length-3 word count $A_{3}$, but, nevertheless, a larger value for the rank criterion than the alternative designs with the same numbers of factors. The nonzero $A_{3}$ value of the $W_{2}$ optimal designs can be recognized in in panel (a) of Figure 1 by looking at the lower end of the leftmost vertical line for each number of factors: that line does not start at zero. The longest vertical line for each number of factors in the plot always corresponds to the $W_{2}^{-}$ optimal design. This is because that design maximizes the confounding of the two-factor interaction effects with the blocks, as opposed to the $W_{2}$ and $W_{3}$ optimal designs.

Another pattern that is visible in the figure is that the upper ends of the vertical lines increase with the number of factors. This shows that, for a given number of experimental runs and a given criterion, studying larger numbers of treatment factors results in larger degrees of aliasing of two-factor interactions and/or confounding of the two-factor interactions with the blocks. Finally, for the case of 11 factors, there exists one design that simultaneously optimizes the $W_{2}^{-}$and $W_{3}$ criteria. For the case of 12 treatment factors, there is a single blocking arrangement that is optimal in terms of all three criteria. The treatment design in that blocking arrangement is a folded-over 12-run Plackett-Burman design. Each block in the blocking arrangement consists of four specific mirror image pairs.

Panel (b) of Figure 1 shows the results for 24-run designs involving 13-16 factors and three blocks. For these numbers of factors, no strength-3 designs exist and the rank of the interaction model matrix equals 24 for all designs under study. Therefore, for these numbers of factors, the $W_{2}$ and $W_{2}^{-}$criteria are more alike than for smaller numbers of factors, where the rank for strength-3 designs is always lower than that for the best strength-2 designs. More specifically, for 13-16 factors, the $W_{2}$ and $W_{2}^{-}$criteria favor the same two-level treatment designs. For the case of 13 factors, there is only one way to block that treatment design, so that the $W_{2}$ and $W_{2}^{-}$optimal blocking arrangements are the same. The treatment design has a maximum absolute type- 3 correlation of $2 / 3$ and an $A_{3}$ value of 6 , while the $A_{3}^{(b t o t)}$ value equals 12 . In contrast, the $W_{3}$ optimal blocking arrangement for 13 factors has a 
treatment design with a maximum absolute type-3 correlation of $1 / 3$ only, an $A_{3}$ count of 10 and an $A_{3}^{(b t o t)}$ value of 10 . So, neither of the two blocked designs outperforms the other in all possible ways.

For 14 and 15 factors, the $W_{2}$ and $W_{2}^{-}$optimal blocking arrangements use the same treatment design, but the assignment of the treatments to the blocks differs. The difference between these arrangements is too small to be of practical importance. It is barely visible in the plot. The comparison between the $W_{2}$ and $W_{2}^{-}$optimal designs, on the one hand, and the $W_{3}$ optimal designs, on the other hand, is analogous to that for the 13-factor designs.

Finally, the selected 16-factor blocking arrangements are either $W_{2}$ and $W_{2}^{-}$optimal or $W_{3}$ optimal. Both involve a two-level treatment design with absolute type-3 correlations of $2 / 3$. There is very little practical difference between these blocking arrangements.

\subsection{4-run designs in four blocks}

Panel (c) of Figure 1 shows the results for 24-run treatment designs involving 4-12 treatment factors and four blocks. The salient features of these designs strongly resemble those of the designs arranged in three blocks. For example, the blocking arrangements that are optimal in terms of the $W_{2}^{-}$and $W_{3}$ criteria also have a strength-3 treatment design (and thus a zero $A_{3}$ value). The plot in panel (c) also shows that, all other things being equal, it is harder to achieve good blocking arrangements for four blocks of size 6 than for three blocks of size 8. As a matter of fact, the upper ends of the vertical lines in the figure's panel (c) are higher than those in panel (a). In other words, the total generalized length-3 word count, $A_{3}+A_{3}^{(b t o t)}$, measuring the confounding between the two-factor interactions and the blocks plus the aliasing between main effects and two-factor interactions, is larger.

Panel (d) of Figure 1 shows the results for 24-run designs involving 13-20 treatment factors and arranged in four blocks. The most striking feature of the plot is that, for each given number of factors, the optimal blocking arrangements have the same generalized length-3 word count $A_{3}$. Indeed, except for 15 factors, the optimal blocking arrangements 
Table 6: Characteristics of the $W_{2}, W_{2}^{-}$and $W_{3}$ optimal blocking arrangements of 15 -factor 24-run treatment designs in four blocks. The elements of the $F A_{3}$ vector correspond to length-3 word counts of $1,5 / 9,3 / 9$ and $1 / 9$.

\begin{tabular}{|c|c|c|c|c|c|c|c|c|}
\hline & $4,16,8)$ & & 24 , & $6,8)$ & \multicolumn{3}{|c|}{$F A_{3}$} & Optimality \\
\hline 0 & 180 & 2 & 21 & 507 & 1 & 7 & 8 & $W_{2}$ \\
\hline 0 & 180 & 5 & 12 & 516 & 2 & 5 & 9 & $W_{2}^{-}$ \\
\hline 0 & 180 & 2 & 23 & 499 & 0 & 9 & 8 & $W_{3}$ \\
\hline
\end{tabular}

we found only differ in terms of the $F_{4}$ and $F A_{3}$ vectors. The 15 -factor optimal blocking arrangements also differ in terms of the $A_{3}^{(b t o t)}$ value, but the small difference in $A_{3}^{(b t o t)}$ value is hardly visible in the plot.

Table 6 shows the $F_{3}, F_{4}$ and $F A_{3}$ vectors and the block counts $A_{3}^{(b t o t)}$ for the $W_{2}, W_{2}^{-}$ and $W_{3}$ optimal arrangements of 15 -factor 24-run treatment designs in four blocks. The rank of the interaction model matrix is 24 for each of these 15-factor designs. All the designs tabulated possess the smallest $A_{3}$ value possible for a 15-factor design in 24 runs, namely 20. Also, the designs all possess an $A_{4}$ value of 67.67 .

Among all designs with the most attractive $A_{3}$ value and the most attractive $F_{3}$ vector, $F_{3}(24,16,8)=(0,0,180)$, the $W_{2}$ criterion first minimizes the block count $A_{3}^{(b t o t)}$ and the $A_{4}$ value, and then sequentially minimizes the elements of the $F_{4}$ vector. In contrast, among all designs with the most attractive $A_{3}$ value and the most attractive $F_{3}$ vector, the $W_{2}^{-}$criterion first maximizes the block count $A_{3}^{(b t o t)}$ and then sequentially minimizes the $A_{4}$ value and the elements of the $F_{4}$ vector. The minimum value for the block count $A_{3}^{(b t o t)}$ among all designs with the most attractive $A_{3}$ value and $F_{3}$ vector is $157 / 9$, while the maximum value is 159/9. This small difference causes the vertical lines for the $W_{2}$ and $W_{2}^{-}$optimal blocking arrangements in the figure's panel (d) to be nearly equally long. Finally, among all designs with the most attractive $F_{3}$ vector, the $W_{3}$ criterion sequentially minimizes the $F A_{3}$ vector. All these features are listed in Table 6.

When 16, 17 and 19 factors are studied, the $W_{2}$ and $W_{2}^{-}$optimal blocking arrangements are identical, but they differ from the $W_{3}$ optimal blocking arrangement. For 13, 14, 18 and 20 factors, the optimal blocking arrangements all coincide. 


\subsection{4-run designs in six blocks}

Panel (e) of Figure 1 shows the results for 24-run designs involving 4-14 treatment factors and arranged in six blocks. Because of the larger number of blocks, there is more confounding between the two-factor interaction effects and the blocks. Again, the blocking arrangements that are optimal in terms of the $W_{2}^{-}$and $W_{3}$ criteria have a treatment design with a zero $A_{3}$ value when 12 or fewer treatment factors are studied.

The largest number of two-level treatment factors that can be handled with an orthogonal design with 24 runs arranged in six blocks is 14, whereas 16 factors can be studied when there are only three blocks and 20 factors can be studied when there are four blocks. Studying more than 14 treatment factors is possible using a six-block 24-run design, but only with a design that is not orthogonally blocked.

\subsection{8-run designs in seven blocks}

The last series of blocking arrangements we discuss involves 28 runs and seven blocks. Panel (f) of Figure 1 shows the characteristics of the optimal blocking arrangements we identified for that case. The plot shows two different optimal blocking arrangements for four and five factors, three different blocking arrangements for six, eight and nine factors, four different blocking arrangements for seven factors and a single blocking arrangement for 10-12 factors.

The blocking arrangements for seven and eight factors deserve special attention, because these are the only instances we encountered where the $W_{2}$ optimal designs differ from the $W_{1}$ optimal designs. Recall that, in the $W_{2}$ criterion's minimization vector, the block count $A_{3}^{(b t o t)}$ precedes the generalized length- 4 word count $A_{4}$, while, in the $W_{1}$ criterion's minimization vector, the generalized length- 4 word count $A_{4}$ precedes the block count $A_{3}^{(b t o t)}$.

Table 7 shows the rank $R$ of the interaction model matrix, the generalized length- 4 word count $A_{4}$ and the block count $A_{3}^{(b t o t)}$ for the $W_{1}, W_{2}, W_{2}^{-}$and $W_{3}$ optimal 7-factor and 8-factor 28-run designs arranged in seven blocks. All four 7 -factor designs have the same $F_{3}$ vector. Therefore, the $W_{1}$ criterion favors the design with the smallest $A_{4}$ value, while the 
Table 7: Characteristics of the $W_{1}, W_{2}, W_{2}^{-}$and $W_{3}$ optimal arrangements for 28-run 7and 8-factor designs in seven blocks. The elements of the $F A_{3}$ vector correspond to length-3 word counts of $6 / 7,4 / 7$ and $2 / 7$.

\begin{tabular}{cccccccc}
\hline \# factors & $R$ & $A_{4}$ & $A_{3}^{(\text {btot })}$ & \multicolumn{3}{c}{$F A_{3}$} & Optimality \\
\hline 7 & 28 & 1.04 & 6.29 & 0 & 5 & 12 & $W_{1}$ \\
7 & 28 & 1.37 & 5.43 & 0 & 3 & 13 & $W_{2}$ \\
7 & 26 & 2.18 & 9.43 & 3 & 6 & 12 & $W_{2}^{-}$ \\
7 & 28 & 1.53 & 5.43 & 0 & 1 & 17 & $W_{3}$ \\
\hline 8 & 28 & 3.06 & 8.86 & 0 & 8 & 15 & $W_{1}$ \\
8 & 28 & 3.71 & 8.00 & 0 & 4 & 20 & $W_{2}, W_{3}$ \\
8 & 27 & 4.36 & 12.57 & 4 & 8 & 16 & $W_{2}^{-}$ \\
\hline
\end{tabular}

$W_{2}$ criterion favors the design which sequentially minimizes the $A_{3}^{(b t o t)}$ and $A_{4}$ values, the $W_{2}^{-}$criterion favors the design with the largest $A_{3}^{(b t o t)}$ value and ignores the rank, and the $W_{3}$ criterion favors the design with best $F A_{3}$ vector (ignoring the $A_{4}$ value).

For the 8-factor designs, the results are similar to those for the 7-factor designs, although, for that case, the $W_{2}$ and $W_{3}$ criteria produce the same optimal blocking arrangement.

Finally, for nine factors, the $W_{2}$ optimal design has the same generalized length-3 word count $A_{3}$ and the same block count $A_{3}^{(b t o t)}$ as the $W_{3}$ optimal design. The designs differ in the rank of the interaction model matrix, the $A_{4}$ value, the $F_{4}$ vector and the $F A_{3}$ vector.

\subsection{Comparison with the best designs for complete randomization}

Most of the treatment designs that appear in the blocking arrangements that are optimal in terms of the three criteria we study here are also optimal or nearly optimal when run in a completely randomized fashion. Specifically, most of these designs are also best, second best or third best when all possible treatment designs are ordered according to the $F_{3}$ and $F_{4}$ vectors or according to the $\left(A_{3}, F_{3}, A_{4}, F_{4}\right)$ vector (Schoen et al., 2017). The following top-3 treatment designs, however, cannot be orthogonally blocked when three, four, six or seven blocks are desired:

1. Three blocks, 24 runs, $14-16$ factors: all top-3 treatment designs according to the $F_{3}$ and $F_{4}$ vectors. 
2. Four blocks, 24 runs, 13, 14, 17 and 19 factors: all top-3 treatment designs according to the $F_{3}$ and $F_{4}$ vectors.

3. Four blocks, 24 runs, 15 and 16 factors: all top-3 treatment designs in terms of the $\left(A_{3}, F_{3}, A_{4}, F_{4}\right)$ vector.

4. Six blocks, 24 runs, 14 factors: all top-3 treatment designs according to the $F_{3}$ and $F_{4}$ vectors.

5. Seven blocks, 28 runs, 7 and 8 factors: the best treatment design according to the $F_{3}$ and $F_{4}$ vectors.

6. Seven blocks, 28 runs, 9-12 factors: all top-3 treatment designs according to the $F_{3}$ and $F_{4}$ vectors.

\section{$5 \quad$ Two practical examples}

In this section, we present two examples from our own consulting experience that required the arrangement of 24-run designs in three or four blocks. Both examples involved 12 twolevel treatment factors. As there is only one optimal design for both these cases, they were not suitable to introduce the different criteria in the beginning of the paper.

\subsection{The cytotoxicity experiment}

The first example deals with the detection of influential factors in a protocol to determine the so-called $\mathrm{EC}_{50}$ value of water pollutants. The $\mathrm{EC}_{50}$ value is the pollutant concentration that causes $50 \%$ of the cells in an in vitro cell culture to die. There was a concern that seemingly

innocuous changes in the protocol might result in substantially different outcomes. We investigated the effect of 12 protocol features on the $\mathrm{EC}_{50}$ value using a folded over 12-run Plackett-Burman design. This design has a strength of 3 and a rank of the interaction model matrix equal to 24 . Obviously, the 12 protocol features correspond to the 12 factors used 
Table 8: Factor names for the cytotoxicity and car tire experiments.

\begin{tabular}{lll}
\hline factor & cytotoxicity & car tire \\
\hline X1 & number of wells & rubber compound \\
X2 & seeding density & number of ribs \\
X3 & seeding time & shoulder block position \\
X4 & dosing & center line cut depth \\
X5 & medium & center line cut width \\
X6 & serum & shoulder cut width \\
X7 & concentration & center line cut angle \\
X8 & solvent & shoulder cut angle \\
X9 & volume & center line cut through \\
X10 & shaking & shoulder cut through \\
X11 & repeated dosing & center line additional sipe \\
X12 & exposure time & shoulder additional sipe \\
\hline
\end{tabular}

in the experiment. The names of the 12 factors in the cytotoxicity experiment are listed in Table 8. A single run required several days of work, but it was possible to perform eight runs in parallel. Therefore, three blocks of eight runs were required to complete the experiment.

Table 5 shows that there are 3,305,030 different designs with one three-level (blocking) factor and 12 two-level (treatment) factors in 24 runs. Three of these involve the folded-over Plackett-Burman design for the treatments. So, there exist three nonisomorphic blocking arrangements of the folded over Plackett-Burman design. The $A_{3}, A_{4}$ and $A_{3}^{(b t o t)}$ values and the $F_{3}$ and $F_{4}$ vectors of these three blocking arrangements are the same, but the arrangements differ in their $F A_{3}$ vectors. More specifically, the $F A_{3}$ vectors of the three blocking arrangements equal $(0,12,36),(3,6,42)$ and $(6,0,48)$, where the entries of the $F A_{3}$ vectors correspond to word counts of $2 / 3,1 / 2$ and $1 / 6$. Since the three options only differ in their $F A_{3}$ vector, the blocking arrangement with the most attractive $F A_{3}$ vector is the best of the three. Clearly, this is the blocking arrangement with the $F A_{3}$ vector equal to $(0,12,36)$.

The folded-over Plackett-Burman design and the blocking arrangement producing an $F A_{3}$ vector equal to $(0,12,36)$ are shown in the first 13 columns of Table 9 . The initial 12 columns contain the levels of the treatment factors, while the 13th column shows the 
Table 9: $W_{2}, W_{2}^{-}$and $W_{3}$ optimal arrangements of a folded over 12-run Plackett-Burman design in three and in four blocks, with $\mathrm{EC}_{50}$ response values from a cytotoxicity experiment.

\begin{tabular}{ccccccccccccccc}
\hline $\mathrm{X} 1$ & $\mathrm{X} 2$ & $\mathrm{X} 3$ & $\mathrm{X} 4$ & $\mathrm{X} 5$ & $\mathrm{X} 6$ & $\mathrm{X} 7$ & $\mathrm{X} 8$ & $\mathrm{X} 9$ & $\mathrm{X} 10$ & $\mathrm{X} 11$ & $\mathrm{X} 12$ & $\mathrm{~B} 1$ & $\mathrm{EC}_{50}$ & $\mathrm{~B} 2$ \\
\hline- & - & - & - & - & - & - & - & - & - & - & - & 3 & 1.377 & 1 \\
- & - & - & - & - & - & + & + & + & + & + & + & 2 & 8.241 & 1 \\
- & - & - & + & + & + & - & - & - & + & + & + & 1 & 0.1875 & 1 \\
- & - & + & - & + & + & - & + & + & - & - & + & 1 & 0.1209 & 2 \\
- & - & + & + & - & + & + & - & + & - & + & - & 3 & 0.4063 & 2 \\
- & - & + & + & + & - & + & + & - & + & - & - & 3 & 1.665 & 3 \\
- & + & - & - & + & + & + & + & - & - & + & - & 1 & 1.32 & 2 \\
- & + & - & + & - & + & - & + & + & + & - & - & 2 & 0.915 & 3 \\
- & + & - & + & + & - & + & - & + & - & - & + & 2 & 0.3267 & 4 \\
- & + & + & - & - & + & + & - & - & + & - & + & 1 & 0.0481 & 3 \\
- & + & + & - & + & - & - & - & + & + & + & - & 2 & 3.016 & 4 \\
- & + & + & + & - & - & - & + & - & - & + & + & 3 & 1.722 & 4 \\
+ & - & - & - & + & + & + & - & + & + & - & - & 3 & 0.01179 & 4 \\
+ & - & - & + & - & + & + & + & - & - & - & + & 2 & 0.2809 & 4 \\
+ & - & - & + & + & - & - & + & + & - & + & - & 1 & 0.4971 & 3 \\
+ & - & + & - & - & + & - & + & - & + & + & - & 2 & 4.438 & 4 \\
+ & - & + & - & + & - & + & - & - & - & + & + & 2 & 0.6222 & 3 \\
+ & - & + & + & - & - & - & - & + & + & - & + & 1 & 0.2552 & 2 \\
+ & + & - & - & - & + & - & - & + & - & + & + & 3 & 0.3211 & 3 \\
+ & + & - & - & + & - & - & + & - & + & - & + & 3 & 0.821 & 2 \\
+ & + & - & + & - & - & + & - & - & + & + & - & 1 & 1.572 & 2 \\
+ & + & + & - & - & - & + & + & + & - & - & - & 1 & 0.5872 & 1 \\
+ & + & + & + & + & + & - & - & - & - & - & - & 2 & 1.538 & 1 \\
+ & + & + & + & + & + & + & + & + & + & + & + & 3 & 0.1469 & 1 \\
\hline
\end{tabular}

assignment of the treatments to the three blocks. The blocking arrangement was actually used for the cytotoxicity experiment. The responses are given in the 14th column of the table. Among the 3,305,030 possible orthogonal blocking arrangements for the cytotoxicity experiment, no better options exist than the one based on the folded over Plackett-Burman design and shown in Table 9 . So, that blocking arrangement is $W_{2}, W_{2}^{-}$and $W_{3}$ optimal.

In $W_{2}, W_{2}^{-}$and $W_{3}$ optimal blocking arrangements, the main effects are orthogonal to the block effects, because of the orthogonal blocking. They are orthogonal to the two-factor interactions as well, because the treatment design in the cytotoxicity experiment is a foldover design. So, the treatment design is a strength-3 design. Consequently, a sensible way to start the data analysis is to identify significant main effects first. The second step in the 
analysis could then be to assume weak effect heredity (Wu and Hamada, 2009) and explore all two-factor interactions which involve at least one factor with a significant main effect. This kind of strategy is recommended by Miller and Sitter (2001).

As the largest response value differs from the smallest one by a factor of about 700, we used a logarithmic transformation of the response values in the data analysis. Figure 2 shows the absolute values of the main effects obtained when using base-10 logarithms. The main effects of the factors exposure time (X12), solvent (X8) and serum (X6) stand out. The second stage of the analysis suggested that the interaction of the factor dosing (X4) with both solvent and serum are probably active.

\subsection{The car tire experiment}

Our second practical example is concerned with the wear of car tires. As the cytotoxicity experiment, the car tire experiment involved 12 two-level treatment factors and the total number of experimental runs was 24 . The names of the factors are listed in the last column of Table 8. The difference between the cytotoxicity experiment and the car tire experiment is that the latter required four blocks of six runs, rather than three blocks of eight runs. This is due to the fact that the experimenters could only test six different tires per day and substantial day-to-day variation was anticipated.

The design actually used by the experimenters was a replicated 12-run Plackett-Burman design for 11 factors. The 12th factor was set at one level for the first replicate and at the other level for the second replicate. As the experimenters were unaware of the possibility to

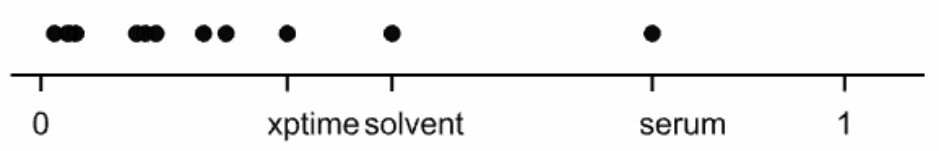

Figure 2: Absolute values of the main effects in the cytotoxicity experiment. 
create an orthogonal blocking arrangement for a 24-run design with six runs per block, they randomly assigned the 24 runs to four blocks. The result is that, in their design, the main effects of the 12 factors were not orthogonal to the blocks. Due to the fact that the 12-run Plackett-Burman design was replicated rather than folded over, the main effects of the 11 treatment factors accommodated by that design were partially aliased with the two-factor interactions. That is not the case for the main effect of the 12 th treatment factor.

The treatment design we would have recommended for the car tire experiment is the one in Table 9. So, as for the cytotoxicity experiment, it is best to use a folded over 12-run Plackett-Burman design. The challenge then is to find an optimal arrangement for that design in four blocks of six runs.

Table 5 shows that there are 12,096,092 different orthogonal blocking arrangements with one four-level (blocking) factor and 12 two-level (treatment) factors in 24 runs. Four of these involve the folded-over Plackett-Burman design for the treatment factors. The $A_{3}, A_{4}$ and $A_{3}^{(b t o t)}$ values and the $F_{3}$ and $F_{4}$ vectors are the same for these four blocking arrangements. Any of the four designs is therefore $W_{2}$ and $W_{2}^{-}$optimal. However, the four designs differ in their $F A_{3}$ vectors. For this case, there are two blocking arrangements that sequentially minimize the $F A_{3}$ vector. The optimal entries are 18, 12 and 36 , corresponding to $A_{3}^{(b 22)}$ values of $5 / 9,1 / 3$ and $1 / 9$, respectively. So, these two blocking arrangements are $W_{2}, W_{2}^{-}$ and $W_{3}$ optimal. The final column of Table 9 shows how the runs of the folded-over PlackettBurman design are assigned to the four blocks in one of the optimal arrangements.

\section{Discussion}

This paper makes a large number of orthogonal blocking arrangements available for orthogonal two-level designs involving 24 and 28 runs. To identify optimal blocking arrangements, we explored complete catalogs of orthogonal designs with several two-level treatment factors and one multi-level blocking factor. We focused on orthogonal blocking arrangements of orthogonal treatment designs, because these designs result in maximum precision for the 
main effect estimates when estimating a model including the block effects and the treatment factors' main effects only. Depending on the specific case, when using the designs we present for estimating a main effects model, the main effect estimates are either not at all biased when one or more interactions are active, or only to a small extent.

For some of the cases considered here, the restriction to orthogonal blocking comes at a price. Table 5 showed that orthogonal blocking arrangements of 24-run orthogonal designs involving more than 16 two-level factors do not exist if three blocks are desired. Similarly, orthogonal blocking arrangements of the 24-run orthogonal designs involving more than 14 two-level factors do not exist if six blocks are desired, and orthogonal blocking arrangements of the 28-run orthogonal designs involving more than 12 two-level factors do not exist for any number of blocks. Also, a comparison of the results presented here with those in Schoen et al. (2017) for two-level designs with 24 or 28 runs in the absence of blocking revealed a few cases where orthogonal blocking was only feasible for suboptimal treatment designs.

It is, of course, also possible to create blocking arrangements that are not orthogonally blocked, using principles from optimal experimental design (Goos and Jones, 2011). We would welcome follow-up research investigating the capabilities of nonorthogonal blocking of good treatment designs with 24 and 28 runs. One aspect of this follow-up research could be to develop a simulation tool to study the power for detecting active main effects and two-factor interactions in blocked experiments. This would provide an objective basis for comparing orthogonally blocked $\left(W_{2}, W_{2}^{-}\right.$and $W_{3}$ optimal) and nonorthogonally blocked designs.

\section{Supplementary materials}

Tables.pdf: tables showing the $F_{3}, F_{4}$ and $F A_{3}$ vectors along with the ranks of the interaction model matrices for the optimal designs.

Designs.zip: all optimal orthogonally blocked designs with four or more treatment fac- 
tors.

\section{Acknowledgements}

This research was financially supported by the Flemish Foundation for Scientific Research FWO-Vlaanderen. The cytotoxicity experiment comes from a collaboration of the first author with Dr Nynke Kramer from the Toxicology Division of the Institute for Risk Assessment Sciences (IRAS) at Utrecht University in the Netherlands.

\section{References}

Bisgaard, S. (1994). A note on the definition of resolution for blocked $2^{k-p}$ designs. Technometrics, 36:308-311.

Bulutoglu, D. A. and Ryan, K. J. (2015). Algorithms for finding generalized minimum aberration designs. Journal of Complexity, 31:577-589.

Cheng, C. S., Mee, R. W., and Yee, O. (2008). Second order saturated orthogonal arrays of strength three. Statistica Sinica, 18:105-119.

Cheng, S. W., Li, W., and Ye, K. Q. (2004). Blocked nonregular two-level factorial designs. Technometrics, 46:269-279.

Cheng, S. W. and Wu, C. F. J. (2002). Choice of optimal blocking schemes in two-level and three-level designs. Technometrics, 44:269-277.

Deng, L. Y. and Tang, B. (1999). Generalized resolution and minimum aberration criteria for Plackett-Burman and other nonregular factorial designs. Statistica Sinica, 9:1071-1082.

Deng, L. Y. and Tang, B. (2002). Design selection and classification for Hadamard matrices using generalized minimum aberration criteria. Technometrics, 44:173-184. 
Goos, P. and Jones, B. (2011). Optimal design of experiments: a case study approach. Wiley, Chichester, UK.

Li, Y., Deng, L. Y., and Tang, B. (2004). Design catalog based on minimum G-aberration. Journal of Statistical Planning and Inference, 124:219-230.

Miller, A. and Sitter, R. R. (2001). Using the folded-over 12-run Plackett-Burman design to consider interactions. Technometrics, 43:44-55.

Montgomery, D. C. (2009). Design and analysis of experiments. 7th edition, Wiley, New York.

National Bureau of Standards (1957). Fractional factorial designs for factors at two levels. Applied Mathematics Series 48; Washington DC: US Government Printing Office.

Schoen, E. D. (2012). Designs for two-stage intensive screening of pharmaceutical production processes. Statistics in Biopharmaceutical Research, 4:336-347.

Schoen, E. D., Eendebak, P. T., and Nguyen, M. V. M. (2010). Complete enumeration of pure-level and mixed-level orthogonal arrays. Journal of Combinatorial Designs, 18:123140.

Schoen, E. D. and Mee, R. W. (2012). Two-level designs of strength 3 and up to 48 runs. Journal of the Royal Statistical Society Series C, 61:163-174.

Schoen, E. D., Sartono, B., and Goos, P. (2013). Optimum blocking for general resolution-3 designs. Journal of Quality Technology, 45:166-187.

Schoen, E. D., Vo-Thanh, N., and Goos, P. (2017). Two-level orthogonal screening designs with 24, 28, 32 and 36 runs. Journal of the American Statistical Association. DOI 10.1080/01621459.2017.1279547.

Sitter, R. R., Chen, J., and Feder, M. (1997). Fractional resolution and minimum aberration in blocked $2^{n-k}$ designs. Technometrics, 39:382-390. 
Sun, D. X., Li, W., and Ye, K. Q. (2008). An algorithm for sequentially constructing nonisomorphic orthogonal designs and its applications. Statistics and Applications, 6:144158.

Sun, D. X., Wu, C. F. J., and Chen, Y. (1997). Optimal blocking schemes for $2^{n}$ and $2^{n-p}$ designs. Technometrics, 39:298-307.

Tang, B. and Deng, L. Y. (1999). Minimum $\mathrm{G}_{2}$-aberration for nonregular fractional factorial designs. Annals of Statistics, 27:1914-1926.

Wu, C. F. J. and Hamada, M. S. (2009). Experiments: Planning, Analysis, and Parameter Design Optimization. 2nd edition, Wiley, New York, NY, USA.

$\mathrm{Xu}, \mathrm{H}$. (2006). Blocked regular fractional factorial designs with minimum aberration. Annals of Statistics, 34:2534-2553.

Xu, H. and Deng, L. Y. (2005). Moment aberration projection for nonregular fractional factorial designs. Technometrics, 47:121-131.

Xu, H. and Wu, C. F. J. (2001). Generalized minimum aberration for asymmetrical fractional factorial designs. Annals of Statistics, 29:1066-1077. 